\title{
Retropharyngeal Neoplasm
}

National Cancer Institute

\section{Source}

National Cancer Institute. Retropharyngeal Neoplasm. NCI Thesaurus. Code C162825.

A benign or malignant neoplasm that affects the retropharyngeal space. 